\title{
Non-Born-Oppenheimer calculations of atoms and molecules
}

\author{
Mauricio Cafiero, Sergiy Bubin and Ludwik Adamowicz \\ Department of Chemistry, University of Arizona, Tucson, Arizona 85721, USA
}

Received 14th November 2002, Accepted 25th February 2003
First published as an Advance Article on the web 5th March 2003

We review a recent development in high-accuracy non-Born-Oppenheimer calculations of atomic and molecular systems in a basis of explicitly correlated Gaussian functions. Much of the recent progress in this area is due to the derivation and implementation of analytical gradients of the energy functional with respect to variational linear and non-linear parameters of the basis functions. This method has been used to obtain atomic and molecular ground and excited state energies and the corresponding wave functions with accuracy that exceeds previous calculations. Further, we have performed the first calculations of non-linear electrical properties of molecules without the Born-Oppenheimer approximation for systems with more than one electron. The results for the dipole moments of such systems as HD and LiH agree very well with experiment. After reviewing our non-Born-Oppenheimer results we will discuss ways this method can be extended to deal with larger molecular systems with and without an external perturbation.

\section{Introduction}

The vast majority of quantum chemical calculations done today and in fact for the last several decades are performed within the Born-Oppenheimer (BO) approximation. In this approximation the electronic part of the wave function is solved for arbitrary arrangements of stationary nuclei, and the nuclear wave function is found for a potential energy surface due to the electrons. This separation of nuclear and electronic motion is acceptable in most calculations of molecular properties and reaction dynamics (a rule of thumb is that the importance of the $\mathrm{BO}$ corrections to electronic values are weighted by $m_{e} / M$ where $m_{e}$ is the mass of an electron and $M$ is the mass of the lightest nucleus in the molecule of interest). For the most accurate calculations, though, and in order to model situations in which the $\mathrm{BO}$ approximation breaks down, non-BO calculations must be performed.

In a recent paper, Handy and $\mathrm{Lee}^{1}$ evaluated the importance of diagonal non-BO corrections to electronic values of quantities such as harmonic frequencies and molecular geometries. They found that the corrections were negligible for most molecules, but more important for molecules containing hydrogen atoms. It is believed that molecules containing loosely bound hydrogens/protons will have significant non-BO corrections.

There has been a recent increase in interest in the nonadiabatic effects in general. This interest has been motivated in part by the maturation of the fields of ultrafast spectroscopy and molecular dynamics. In experimental studies in these areas there is a higher chance of BO breakdown and observation of nonadiabatic effects. Some of the recent theoretical developments in non-BO methods include self-consistent-field (SCF) approaches in which the total wave function is the product of a determinant for the electrons and a determinant (or a permanent, for bosons) for the nuclei ${ }^{2,3}$ as well as configuration interaction and multi-configurational self-consistent-field methods for the electrons and nuclei based on the abovementioned SCF methods. ${ }^{3,4}$

Work on the non-BO methods has been carried out in our laboratory for the last decade. The centerpiece of this work has been the use of $n$-particle correlated Gaussian functions that explicitly depend on the inter-particular distances, i.e. distances between the electrons, electrons and nuclei, and between nuclei. An important development in the nonadiabatic methods has been the derivation and implementation of the analytical gradient of the variational energy functional with respect to the non-linear exponential parameters of the explicitly correlated Gaussian functions. This method has allowed us to expand the non-BO wave function in a basis of $n$-particle functions and very effectively minimize the resulting expression for the energy variationally in terms of the nonlinear parameters of the basis functions. The application work performed with the non-BO method has included a new upper bound to the nonadiabatic energy of $\mathrm{H}_{2}{ }^{5}$ and its isotopomers ${ }^{6}$ and an upper bound to the ground state energy of $\mathrm{LiH}^{7}$ Some of the more recent work has included development of a method for calculating electrical properties of molecules nonadiabatically. The first calculations performed using this method were of the dipole moments and polarizabilities of $\mathrm{LiH}$ and $\mathrm{LiD}^{8,9}$ and dipole moments, polarizabilities, and first and second hyperpolarizabilities of the $\mathrm{H}_{2}$ isotopomer series. ${ }^{10}$ Where experimental values of the calculated properties exist (dipole moments of $\mathrm{LiH}, \mathrm{LiD}$, and $\mathrm{HD}$ ) the theoretical values agree well. ${ }^{11,12}$ The work on the non-BO non-linear optical properties is the first in the field that considers molecules with more than one electron. ${ }^{13,14}$ Some other previous work in fully nonadiabatic calculations has been done by Kozlowski and Adamowicz, ${ }^{15-25}$ Kinghorn, ${ }^{26}$ and Kinghorn and Poshusta. ${ }^{27}$

There has been much interest recently in the area of electrical and optical properties of molecules which has been well documented and will not be discussed here. One aspect of the theoretical studies on optical properties that is quite relevant to the work reviewed here is the calculation of the nuclear contributions to these properties. In the traditional BornOppenheimer (BO) approximation (which by definition includes the adiabatic approximation) the calculation of the electrical and nuclear wave functions are performed sequentially rather than simultaneously, and thus any properties which are calculated from the wave function, or, equivalently, from the energy of the system, must be done sequentially as well. In many cases calculation of electronic contributions to optical properties are sufficient. In other cases, though, the nuclear contributions are non-negligible and it is necessary 
to include them in order for the result to be quantitatively correct. While methods for calculating electronic properties are well developed, the methods for calculating the nuclear contribution are less well known. Some very good reviews on the topic may be found in the literature. ${ }^{28-30}$

Two of the current authors (Cafiero and Adamowicz) recently collaborated on a study of the magnitude of nonadiabatic effects on the calculations of dipole moments and polarizabilities of some $\mathrm{LiH}$ isotopomers. ${ }^{31}$ It was found that the effects on these properties is quite small, and, in fact was smaller than the assumed errors in the final numbers. A study is currently being made on the magnitude of the nonadiabatic effects on the calculations of the first and second hyperpolarizabilities, where the magnitude may be larger.

Fully non-adiabatic calculations are important for several reasons. As basic physics, they represent an advance in technology for calculating stationary states of atoms and molecules with very high accuracy. Though the theory is basic quantum mechanics, in the past it has only been applied in specialized codes to perform non-adiabatic calculations for one- and two-electron systems of a certain symmetry. ${ }^{13,14}$ The work discussed here has been done with general $n$-particle codes which include very flexible single-center and multi-center explicitly correlated Gaussian basis functions (ECG's). These functions can form complete sets and, as the numerical results indicate, in the complete limit can be used to describe atomic and molecular systems very accurately. It should be noted that the SCF-based methods for calculating the coupled motion of the nuclei and electrons mentioned above depend on a finite orbital expansion in addition to the configuration expansions. While such an approach is typically much less expensive than the method described here, the current method has a capacity for much higher accuracy wave functions due to use of explicitly correlated basis functions. As computational chemistry moves forward and the computational capabilities increase the old emphasis on inexpensive methods will not be as strong and the non-BO method may become the method of choice for problems where high accuracy is desired.

On a more practical note, the current non-BO method is important in that it provides means of verifying the accuracy of the BO-correction methods which add the non-adiabatic corrections due to nuclear motion back to the purely electronic properties using perturbation theory. One example of such a procedure will be discussed below. There are also cases in which the perturbation method to account for non-adiabatic effects are known to fail and in such cases the current method is the only reliable one.

\section{Non-relativistic Hamiltonian}

A system of $n+1$ particles of masses $M_{i}$ and charges $Q_{i}$ may be described at any point in time by the $n+1$ three-dimensional vectors, $\boldsymbol{R}_{i}$, of Cartesian coordinates in the laboratory coordinate system:

$$
\boldsymbol{R}_{i}=\left(\begin{array}{c}
x_{i} \\
y_{i} \\
z_{i}
\end{array}\right)
$$

and the $n+1$ vectors, $\boldsymbol{P}_{i}$, describing the momenta of the particles:

$$
\boldsymbol{P}_{i}=\left(\begin{array}{c}
P_{x, i} \\
P_{y, i} \\
P_{z, i}
\end{array}\right)
$$

For convenience we collect the $\boldsymbol{R}_{i}$ and $\boldsymbol{P}_{i}$ vectors together in vectors $\boldsymbol{R}$ and $\boldsymbol{P}$, respectively:

$$
\boldsymbol{R}=\left(\begin{array}{c}
\boldsymbol{R}_{1} \\
\boldsymbol{R}_{2} \\
\vdots \\
\boldsymbol{R}_{n+1}
\end{array}\right), \quad \boldsymbol{P}=\left(\begin{array}{c}
\boldsymbol{P}_{1} \\
\boldsymbol{P}_{2} \\
\vdots \\
\boldsymbol{P}_{n+1}
\end{array}\right)
$$

The kinetic energy of this system is given by:

$$
T=\sum_{i=1}^{n+1} \frac{\boldsymbol{P}_{i}^{2}}{2 M_{i}} .
$$

If we assume only Coulombic interactions between the particles, the potential energy is given by

$$
V=\sum_{i=1}^{n+1} \sum_{j>i}^{n+1} \frac{Q_{i} Q_{j}}{R_{i j}}
$$

where $R_{i j}$ is the magnitude of the distance vector between particles $i$ and $j: R_{i j}=\left|\boldsymbol{R}_{i}-\boldsymbol{R}_{j}\right|$. The total Hamiltonian for this system is thus:

$$
H_{\mathrm{tot}}=\sum_{i=1}^{n+1} \frac{\boldsymbol{P}_{i}^{2}}{2 M_{i}}+\sum_{i=1}^{n+1} \sum_{j>i}^{n+1} \frac{Q_{i} Q_{j}}{R_{i j}} .
$$

We transform this to the quantum mechanical Hamiltonian operator by substitution of the configuration space operators and we get (atomic units are being use throughout this work):

$$
\hat{H}_{\mathrm{tot}}=\sum_{i=1}^{n+1} \frac{-1}{2 M_{i}} \nabla_{i}^{2}+\sum_{i=1}^{n+1} \sum_{j>i}^{n+1} \frac{Q_{i} Q_{j}}{R_{i j}} .
$$

The interaction of matter and light calls for treating the light quantum mechanically, or as photons. It is often sufficient, though, to treat the matter quantum mechanically and the light classically; this is the semi-classical approach. The light, then, is treated as perpendicular oscillating electric and magnetic fields. The effect of the magnetic portion on matter is usually less than the effect of the electric portion, and so for the work presented here we may neglect the magnetic portion. If we further assume a static field, then we may express the interaction as the scalar product of the total dipole moment of the system and the field vector:

$$
E=-\boldsymbol{\mu} \cdot \boldsymbol{\varepsilon} .
$$

For a derivation of the above Hamiltonian, please see Ratner and Schatz. ${ }^{32}$ We may also express the total quantum mechanical operator as:

$$
\boldsymbol{\mu} \cdot \boldsymbol{\varepsilon}=\sum_{i=1}^{n+1} \boldsymbol{\varepsilon} \cdot \boldsymbol{R}_{i} Q_{i},
$$

where each term in the sum is the interaction of each particle with the electric field. The Hamiltonian for the system in an electric field is thus:

$$
\hat{H}_{\mathrm{tot}}=\sum_{i=1}^{n+1} \frac{P_{i}^{2}}{2 M_{i}}+\sum_{i=1}^{n+1} \sum_{j>1}^{n+1} \frac{Q_{i} Q_{j}}{R_{i j}}-\sum_{i=1}^{n+1} \boldsymbol{\varepsilon} \cdot \boldsymbol{R}_{i} Q_{i} .
$$

The number of degrees of freedom for any system may be reduced by three by a transformation to a new set of coordinates where three coordinates desribe the center-of-mass. In the present work, the system of $n+1$ particles with $3(n+1)$ degrees of freedom is reduced to $n$ pseudo-particles (each with reduced mass $m_{i}$ ) with $3 n$ degrees of freedom with the three remaining degrees of freedom describing the motion of the center of mass.

One such transformation which acts on the vector of coordinates $\boldsymbol{R}$ described above has the following form:

$$
\boldsymbol{T}=\left(\begin{array}{cccccc}
\frac{M_{1}}{M_{T}} & \frac{M_{2}}{M_{T}} & \frac{M_{3}}{M_{T}} & \frac{M_{4}}{M_{T}} & \ldots & \frac{M_{n+1}}{M_{T}} \\
-1 & 1 & 0 & 0 & 0 & \ldots \\
-1 & 0 & 1 & 0 & 0 & \ldots \\
-1 & 0 & 0 & 1 & 0 & \ldots \\
-1 & 0 & 0 & 0 & 1 & \ldots \\
\ldots & \ldots & \ldots & \ldots & \ldots & \ldots
\end{array}\right) \otimes \boldsymbol{I}_{3}
$$


where $M_{\mathrm{T}}$ is the sum of all $n+1$ masses in the original system (i.e., the total mass of the system), $\boldsymbol{I}_{3}$ is the $3 \times 3$ identity matrix, and in general, $\boldsymbol{I}_{n}$ is the $n \times n$ identity matrix, and $\otimes$ denotes the Kronecker product. $\boldsymbol{T}$ transforms $\boldsymbol{R}$, the set of the $3(n+1)$ coordinates in the laboratory coordinate system into $\boldsymbol{R}_{0}$, the set of coordinates describing the position of the center of mass, and $\boldsymbol{R}$, the set of $3 n$ coordinates describing the positions of particle 2 , particle $3, \ldots$, particle $n+1$ with respect to particle 1 (the reference particle):

$$
\boldsymbol{T R}=\left(\begin{array}{c}
\boldsymbol{r}_{0} \\
\boldsymbol{r}_{1} \\
\vdots \\
\boldsymbol{r}_{n}
\end{array}\right)=\left(\begin{array}{c}
\boldsymbol{r}_{0} \\
\boldsymbol{r}
\end{array}\right),
$$

where $\boldsymbol{r}$ is defined implicitly in the above equation.

The momenta are transformed by the inverse transformation:

$$
\boldsymbol{T}^{-1} \boldsymbol{P}=\left(\begin{array}{c}
\boldsymbol{p}_{0} \\
\boldsymbol{p}_{1} \\
\vdots \\
\boldsymbol{p}_{n}
\end{array}\right)=\left(\begin{array}{c}
\boldsymbol{p}_{0} \\
\boldsymbol{p}
\end{array}\right),
$$

where $\boldsymbol{p}_{0}$ describes the momentum of the center of mass and $\boldsymbol{p}$ describes the momenta of the pseudo-particles. The inverse transformation $\boldsymbol{T}^{-1}$ is given by:

$$
\boldsymbol{T}^{-1}=\left(\begin{array}{cccccc}
1 & -\frac{M_{2}}{M_{T}} & -\frac{M_{3}}{M_{T}} & -\frac{M_{4}}{M_{T}} & \cdots & -\frac{M_{n+1}}{M_{T}} \\
1 & \frac{M_{T}-M_{2}}{M_{T}} & -\frac{M_{3}}{M_{T}} & -\frac{M_{4}}{M_{T}} & -\frac{M_{5}}{M_{T}} & \cdots \\
1 & -\frac{M_{2}}{M_{T}} & -\frac{M_{T}-M_{3}}{M_{T}} & -\frac{M_{4}}{M_{T}} & -\frac{M_{5}}{M_{T}} & \ldots \\
1 & -\frac{M_{2}}{M_{T}} & -\frac{M_{3}}{M_{T}} & -\frac{M_{T}-M_{4}}{M_{T}} & -\frac{M_{5}}{M_{T}} & \ldots \\
1 & -\frac{M_{2}}{M_{T}} & -\frac{M_{3}}{M_{T}} & -\frac{M_{4}}{M_{T}} & \frac{M_{T}-M_{5}}{M_{T}} & \ldots \\
\cdots & \cdots & \cdots & \cdots & \cdots & \cdots
\end{array}\right) \otimes \boldsymbol{I}_{3} .
$$

The charges map directly $Q_{i} \rightarrow q_{i-1}$ with the change on the particle at the center of mass mapping to a central potential.

These transformations of the position and the momentum vectors performed on the total Hamiltonian result in the separation of the Hamiltonian into the center-of-mass Hamiltonian that describes the kinetic energy of the center-of-mass motion and the "internal" Hamiltonian describing the internal state of the system. The internal Hamiltonian is dependent on coordinates of the $n$ pseudo-particles:

$$
\begin{aligned}
\hat{H}= & -\frac{1}{2}\left(\sum_{i=1}^{n} \frac{1}{m_{i}} \nabla_{i}^{2}+\sum_{i \neq j}^{n} \frac{1}{M_{1}} \nabla_{i}^{\prime} \nabla_{j}\right) \\
& +\sum_{i=1}^{n} \frac{q_{0} q_{i}}{r_{i}}+\sum_{i<j}^{n} \frac{q_{i} q_{j}}{r_{i j}}-\sum_{i=1}^{n} \varepsilon \cdot \boldsymbol{r}_{\mathbf{i}} q_{i},
\end{aligned}
$$

where $\left({ }^{\prime}\right)$ denotes transposition.

To provide an illustration of the above transformation let us consider an example of a three-particle system with masses $\left\{M_{1}, M_{2}, M_{3}\right\}$ and charges $\left\{Q_{1}, Q_{2}, Q_{3}\right\}$ interacting under a Coulomb potential. In atomic units the total Hamiltonian of the system is:

$$
\hat{\boldsymbol{H}}_{\mathrm{tot}}=\frac{\boldsymbol{P}_{1}^{2}}{2 M_{1}}+\frac{\boldsymbol{P}_{2}^{2}}{2 M_{2}}+\frac{\boldsymbol{P}_{3}^{2}}{2 M_{3}}+\frac{Q_{1} Q_{2}}{R_{12}}+\frac{Q_{1} Q_{3}}{R_{13}}+\frac{Q_{2} Q_{3}}{R_{23}},
$$

where $\boldsymbol{R}_{i}, \boldsymbol{P}_{i}$ are position and momentum vectors for particle $i$ and $R_{i j}=\left\|\boldsymbol{R}_{i}-\boldsymbol{R}_{j}\right\|$. The coordinate and momentum transformation is given by:

$$
\begin{gathered}
r_{0}=\frac{M_{1} \boldsymbol{R}_{1}+M_{2} \boldsymbol{R}_{2}+M_{3} \boldsymbol{R}_{3}}{m_{0}}, \quad \boldsymbol{p}_{0}=\boldsymbol{P}_{1}+\boldsymbol{P}_{2}+\boldsymbol{P}_{3}, \\
m_{0}=M_{1}+M_{2}+M_{3}, \quad q_{0}=Q_{1}
\end{gathered}
$$

$$
\begin{array}{r}
r_{1}=\boldsymbol{R}_{2}-\boldsymbol{R}_{1}, \quad \boldsymbol{p}_{1}=\boldsymbol{P}_{2}-\frac{M_{2}}{m_{0}} \boldsymbol{p}_{0}, \\
m_{1}=\frac{M_{1}+M_{2}}{M_{1}+M_{2}}, \quad q_{1}=Q_{2}, \\
r_{2}=\boldsymbol{R}_{3}-\boldsymbol{R}_{1}, \quad \boldsymbol{p}_{2}=\boldsymbol{P}_{3}-\frac{M_{3}}{m_{0}} \boldsymbol{p}_{0}, \\
m_{2}=\frac{M_{1}+M_{3}}{M_{1}+M_{3}}, \quad q_{2}=Q_{3}
\end{array}
$$

Using these quantities in the full Hamiltonian, $\hat{H}_{\text {tot }}$, results in its separation into the Hamiltonian describing the kinetic energy of the center of mass motion, $\hat{H}_{\mathrm{cm}}$, and the internal Hamiltonian, $\hat{H}$ :

$$
\hat{H}_{\mathrm{tot}}=\hat{H}_{\mathrm{cm}}+\hat{H}
$$

where

$$
\hat{\boldsymbol{H}}_{\mathrm{cm}}=\frac{\boldsymbol{p}_{0}^{2}}{2 m_{0}}
$$

and

$$
\hat{\boldsymbol{H}}=\frac{\boldsymbol{p}_{1}^{2}}{2 m_{1}}+\frac{\boldsymbol{p}_{2}^{2}}{2 m_{2}}+\frac{\boldsymbol{p}_{1} \cdot \boldsymbol{p}_{2}}{M_{1}}+\frac{q_{0} q_{1}}{r_{1}}+\frac{q_{0} q_{2}}{r_{2}}+\frac{q_{1} q_{2}}{r_{12}} .
$$

The internal Hamiltonian in this form has been used in the calculations described here.

\section{Basis functions for non-BO calculations of small atoms}

In the absence of an external perturbation (e.g., electric field) the internal Hamiltonian, $\hat{H}$, is isotropic (fully spherically symmetric) and, thus, its eigenfunctions should form a basis set for irreducible representations of the rotational group. In particular the ground state wave function should be fully spherically symmetric with respect to any rotation in the 3-D space of the internal coordinates. The basis set used in these calculations must reflect this symmetry of the system. Thus in calculating the ground state of closed shell atoms and atoms with only s-electrons in valence shells, which are not completely occupied, the basis functions should be fully spherically symmetric in terms of rotations with respect to the center of the internal coordinates system.

The non-BO calculations have to be carried out with very high precision since the non-BO effects are typically very small. In order to accomplish such precision the correlated motions of the electrons, the electrons and the nuclei (or nucleus in the atomic case), and the nuclei (in the molecular case) have to be very accurately described in the wave function. Since the electrons, particularly the core electrons, closely follow the nuclei and the nuclei, unlike electrons, stay far apart from each other due to their repulsion and large masses, the electron-nucleus and nucleus-nucleus correlations are much stronger than the electron-electron correlation. The most effective way to describe the inter-particle correlation in the system with Coulombic interactions is to use basis functions that explicitly depend on the inter-particle distances.

The symmetry requirements and the need to very effectively describe the correlation effects have turned our attention to explicitly correlated Gaussian functions as the choice for the basis set in the atomic and molecular non-BO calculations. These functions have been used previously in Born-Oppenheimer calculations to describe the electron correlation in molecular systems using the perturbation theory approach. ${ }^{33-40}$ In those calculations, Gaussian pair functions (geminals), each dependent only on a single inter-electron distance in the exponential factor $\exp \left(-\beta r_{i j}^{2}\right)$, were used. In the non-BO work described here, different forms of the explicitly correlated 
Gaussian functions have been used, each dependent on distances between all of the particles that form the system. In the calculations described here the variational method is used to determine the non-adiabatic ground and excited quantum states describing the coupled motion of the nuclei and the electrons. The application of correlated Gaussian functions in molecular calculations has gained momentum in recent years following some impressively accurate works of Jeziorski, Szalewicz and their coworkers, as well as Rychlewski and his coworkers, on small molecular systems performed with the use of these functions. ${ }^{41-45}$

In the atomic non-BO calculations the s-type explicitly correlated Gaussian functions used have the following form:

$$
\phi_{k}=\exp \left[-\boldsymbol{r}^{\prime}\left(\boldsymbol{A}_{k} \otimes \boldsymbol{I}_{3}\right) \boldsymbol{r}\right] .
$$

The above function is a one-center correlated Gaussian with exponential coefficients forming the symmetric matrix $\boldsymbol{A}_{k} . \phi_{k}$ are rotationally invariant functions as required by the symmetry of the problem; that is, invariant to any orthogonal transformation. To show the invariance let $\boldsymbol{U}$ be any $3 \times 3$ orthogonal matrix (any proper or improper rotation in 3space) that is applied to rotate the $\boldsymbol{r}$ vector in the 3-D space: prove the invariance:

$$
\left(\left(\boldsymbol{I}_{n} \otimes \boldsymbol{U}\right) \boldsymbol{r}\right)^{\prime}\left(\boldsymbol{A}_{k} \otimes \boldsymbol{I}_{3}\right)\left(\boldsymbol{I}_{n} \otimes \boldsymbol{U}\right) \boldsymbol{r}=\boldsymbol{r}^{\prime}\left(\boldsymbol{I}_{n} \otimes \boldsymbol{U}^{\prime}\right)\left(\boldsymbol{A}_{k} \otimes \boldsymbol{I}_{3}\right)\left(\boldsymbol{I}_{n} \otimes \boldsymbol{U}\right) \boldsymbol{r}
$$

$$
\begin{aligned}
& =\boldsymbol{r}^{\prime}\left(\boldsymbol{A}_{k} \otimes \boldsymbol{U}^{\prime} \boldsymbol{U}\right) \boldsymbol{r} \\
& =\boldsymbol{r}^{\prime}\left(\boldsymbol{A}_{k} \otimes \boldsymbol{I}_{3}\right) \boldsymbol{r} .
\end{aligned}
$$

$\phi_{k}$ can be also expressed in the more conventional form used in the electronic structure calculations as:

$$
\begin{aligned}
\phi= & \exp \left[-\alpha_{1 k} r_{1}^{2}-\alpha_{2 k} r_{2}^{2}-\ldots-\alpha_{n k} r_{n}^{2}-\beta_{12, k} r_{12}^{2}\right. \\
& \left.-\beta_{13, k} r_{13}^{2}-\ldots-\beta_{n(n-1), k} r_{n(n-1)}^{2}\right] .
\end{aligned}
$$

In this form the $n$-particle correlated Gaussian is a product of $n$ orbital gaussians and $n(n-1) / 2$ Gaussian pair functions (geminals).

To describe bound stationary states of the system, the $\phi_{k}$ have to be square-normalizable functions. The square-integrability of these functions may be achieved using the following general form of an $n$-particle correlated Gaussian with the negative exponential of a positive definite quadratic form in $3 n$ variables:

$$
\phi_{k}=\exp \left[-\boldsymbol{r}^{\prime}\left(\boldsymbol{L}_{k} \boldsymbol{L}_{k}^{\prime} \otimes \boldsymbol{I}_{3}\right) \boldsymbol{r}\right] .
$$

Here $\boldsymbol{r}$ is a $3 n \times 1$ vector of Cartesian coordinates for the $n$ particles, and $\boldsymbol{L}_{k}$ is an $n \times n$ lower triangular matrix of rank $n$ whose elements may vary in the range $[-\infty, \infty]$.

\section{Permutational symmetry of the wave function}

The energy of a quantum system is invariant to permutations of identical particles in the system. Thus, the Hamiltonian for a system with $n$ identical particles has to commute with the elements of the $n$th order symmetric group:

$$
\left[\hat{H}, S_{n}\right]=0 .
$$

This requires that the eigenfunctions of the Hamiltonian in the basis described in the previous section are simultaneously eigenfunctions of both the Hamiltonian and the symmetric group. This may be accomplished by taking the above mentioned basis functions, which may be called primitive basis functions, and projecting them onto the appropriate irreducible representation of the symmetric group. After this treatment we may call the basis functions symmetry projected basis functions.
The projection operator takes the form of a sum of all of the possible permutations of the identical particles, $\hat{P}_{i}$, each multiplied by an appropriate constant, $\chi_{i}$ :

$$
\hat{\mathscr{P}}=\sum_{i=1}^{n !} \chi_{i} \hat{P}_{i} .
$$

The Pauli antisymmetry principle requires that the wave function (including spin degrees of freedom), and thus the basis functions, for a system of identical particles must transform like the totally antisymmetric irreducible representation in the case of fermions, or spin $\frac{1}{2} k$ particles (where $k$ is odd), and like the totally symmetric irreducible representation in the case of bosons, or spin $k$ particles (where $k$ may take on any integer value).

Projection operators for irreducible representations of the symmetric group are obtained easily from their corresponding Young tableaux. ${ }^{46} \mathrm{~A}$ simple way to do this for small $n$ is described as follows. We define an operator $A$ to be the antisymmetrizer for rows of the Young tableaux:

$$
A=\prod_{i=1}^{\text {rows }} \sum_{i}^{n !} \delta \hat{P}_{i},
$$

where $\delta$ is positive for odd permutations and negative for even permutations. We also define an operator $S$ to be the symmetrizer for columns of the Young tableaux:

$$
S=\prod^{\text {columns }} \sum_{i=1}^{n !} \hat{\boldsymbol{P}}_{i} .
$$

The Young operator, or the projection operator, is then the product $\hat{\mathscr{P}}=A S$.

As a simple example of the application of the permutational symmetry in the explcicitly correlated Gaussian basis set, one may examine the $\mathrm{H}^{-}$system and its isotopomers $\left(\mathrm{D}^{-}\right.$and $\mathrm{T}^{-}$). After separating the center-of-mass motion from the Schrödinger equation, the problem is reduced to a twopseudo-particle problem where the internal coordinate $r$, $\boldsymbol{r}=\left(\boldsymbol{r}_{1}^{\prime}, \boldsymbol{r}_{2}^{\prime}\right)^{\prime}$, is the $6 \times 1$ vector of relative coordinates. In this case the ground state spatial wave function is symmetric with respect to the permutation of the coordinates of the two electrons. This symmetry condition is met using the following symmetrized form of the basis functions:

$\phi_{k}(\boldsymbol{r})=\exp \left[-\boldsymbol{r}^{\prime}\left(\boldsymbol{L}_{k} \boldsymbol{L}_{k}^{\prime} \otimes \boldsymbol{I}_{3}\right) \boldsymbol{r}\right]+\exp \left[-\boldsymbol{r}^{\prime}\left(\boldsymbol{\tau}^{\prime} \boldsymbol{L}_{k} \boldsymbol{L}_{k}^{\prime} \boldsymbol{\tau} \otimes \boldsymbol{I}_{3}\right) \boldsymbol{r}\right]$,

where $\tau$ is the permutation matrix $\left(\begin{array}{ll}0 & 1 \\ 1 & 0\end{array}\right)$.

\section{Variational method}

In our calculations the wave function for the ground state is obtained by directly minimizing the Rayleigh quotient:

$$
E(\boldsymbol{a} ; \boldsymbol{c})=\min _{\{\boldsymbol{a}, \boldsymbol{c}\}} \frac{\boldsymbol{c}^{\prime} \boldsymbol{H}(\boldsymbol{a}) \boldsymbol{c}}{\boldsymbol{c}^{\prime} \boldsymbol{S}(\boldsymbol{a}) \boldsymbol{c}}
$$

with respect to the linear expansion coefficients, $\boldsymbol{c}$, of the wave function in terms of the symmetrized basis functions, and with respect to the non-linear exponential parameters, $\boldsymbol{a}$, of the basis functions. In the ground state calculations diagonalization of the $\boldsymbol{H}$ matrix is usually not performed since it is believed that the energy can be more effectively lowered when the $c$ and $\boldsymbol{a}$ variables are considered independent parameters. In minimizing the energies of the excited states, which has been done in separate calculations for each excited state, we used the diagonalization procedure that provides the eigenvalue only for the calculated root. 
In the minimization of the energy functional the analytical gradient of the energy with respect to the linear and non-linear parameters of the wave function is used. The energy gradient with respect to the non-linear variational parameters can be written as:

$$
\nabla_{\boldsymbol{a}} E=\frac{1}{\boldsymbol{c}^{\prime} \boldsymbol{S} \boldsymbol{c}}\left(\frac{\partial \operatorname{vech} \boldsymbol{H}}{\partial \boldsymbol{a}^{\prime}}-E \frac{\partial \operatorname{vech} \boldsymbol{S}}{\partial \boldsymbol{a}^{\prime}}\right)^{\prime}\left(\operatorname{vech}\left[2 \boldsymbol{c} \boldsymbol{c}^{\prime}-\operatorname{diag} \boldsymbol{c} \boldsymbol{c}^{\prime}\right]\right)
$$

where $\boldsymbol{a}$ is a $m(n(n+1) / 2)$ vector of all the non-linear parameters, $m$ is the number of basis functions used, and the vech operator vectorizes a symmetric matrix by stacking the columns of the lower triangular part of the matrix (excluding the upper triangular part) on top of each other. The vector $\boldsymbol{a}$ is made by stacking, function by function, the lower triangular elements of $\boldsymbol{L}_{k}$. In the case of Gaussians with shifted centers described later in this work, the $\boldsymbol{a}$ vector also includes the elements of the $\boldsymbol{s}_{k}$ shift vector. The matrices of derivatives $\partial$ vech $\boldsymbol{H} / \partial \boldsymbol{a}^{\prime}$ and $\partial$ vech $\boldsymbol{S} / \partial \boldsymbol{a}^{\prime}$ are sparse, since each row has at most $2(n(n+1) / 2)$ non-zero elements $(2(n(n+1) / 2+3 n)$ for shifted Gaussians). The derivatives of the Hamiltonian matrix are determined using the derivatives of the molecular integrals:

$$
\frac{\partial \text { vech } \boldsymbol{H}}{\partial \boldsymbol{a}^{\prime}}=\frac{\partial \operatorname{vech} \boldsymbol{T}}{\partial \boldsymbol{a}^{\prime}}+\frac{\partial \text { vech } \boldsymbol{V}}{\partial \boldsymbol{a}^{\prime}} .
$$

The integrals and their derivatives have been described in the previous papers. ${ }^{6,9,17,20,24}$ The analytical gradients considerably accelerate the variational optimization of the wave function.

\section{An example of atomic non-Born-Oppenheimer calculations}

As an example of very accurate non-BO calculations we present our studies of the electron affinity of $\mathrm{H}, \mathrm{D}$, and $\mathrm{T} .{ }^{47}$ The calculated results and their comparison with the experimental results of Lineberger and coworkers ${ }^{48}$ are shown in Table 1. The calculated results include the relativistic, relativistic recoil, Lamb shift, and finite nuclear size corrections labeled $\Delta E_{\text {corr }}$ calculated by Drake. ${ }^{49}$ As one notices the agreement with the experiment for $\mathrm{H}$ and $\mathrm{D}$ is excellent. The $3.7 \mathrm{~cm}^{-1}$ increase of the electron affinity in going from $H$ to $D$ is very well reproduced in the calculations. Since the electron affinity of $\mathrm{T}$ has not been measured yet, the calculated value may serve as a reference for future experimental attempts to measure this quantity.

\section{Diatomic non-Born-Oppenheimer calculations}

Encouraged by the high accuracy of the $\mathrm{H} / \mathrm{H}^{-}, \mathrm{D} / \mathrm{D}^{-}$, and $\mathrm{T} / \mathrm{T}^{-}$calculations we applied the explicitly correlated Gaussian

Table 1 Electron affinity of hydrogen, deuterium and tritium atoms obtained with 300 explicitly correlated Gaussian functions. The term $\Delta E_{\text {corr }}$ contains relativistic, relativistic recoil, Lamb shift and finite nuclear size corrections. ${ }^{47}$

\begin{tabular}{lccc}
\hline & Hydrogen & Deuterium & Tritium \\
\hline$\left(E_{\mathrm{H}}-E_{\mathrm{H}^{-}}\right) / \mathrm{cm}^{-1}$ & 6083.4058 & 6087.0201 & 6088.2233 \\
$\Delta E_{\mathrm{corr}} / \mathrm{cm}^{-1}($ ref. 49$)$ & 0.307505 & 0.307589 & 0.307616 \\
$\mathrm{EA} / \mathrm{cm}^{-1}$ & 6083.0983 & 6086.7126 & 6087.9157 \\
Lykke et al. & $6082.99 \pm 0.15$ & $6086.2 \pm 0.6$ & - \\
$\quad($ experiment $) / \mathrm{cm}^{-1}$ & & & \\
$\quad($ ref. 48$)$ & & & \\
\hline
\end{tabular}

basis, $\phi_{k}=\exp \left[-\boldsymbol{r}^{\prime}\left(\boldsymbol{L}_{k} \boldsymbol{L}_{k}^{\prime} \otimes \boldsymbol{I}_{3}\right) \boldsymbol{r}\right]$, to the simplest diatomic system $\mathrm{H}_{2}{ }^{+}$. It was believed that since the ground state non-BO $\mathrm{H}_{2}{ }^{+}$wave function like the $\mathrm{H} / \mathrm{H}^{-}$wave functions is spherically symmetric the atomic basis should also work well for the molecular case. However, the first calculations performed led to disappointing results, regardless of the number of basis functions used. The optimization of the non-linear parameters in this case always led to significant linear dependencies in the basis set.

In an attempt to determine the origin of the problem the following was tried: ${ }^{50}$ The very accurate Born-Oppenheimer potential energy curve for the ground state of $\mathrm{H}_{2}$ from the work of Kolos and Wolniewicz ${ }^{51}$ was approximated by a series of spherically symmetric Gaussian functions multiplied by powers of inter-nuclear distance, $r$ :

$$
V(r)=c_{0}+\sum_{i} c_{i} r^{m_{i}} \mathrm{e}^{-b_{i} r^{2}}
$$

The non-BO variational method was then used to determine the ground and excited vibrational states of this system. By replacing the two electrons of $\mathrm{H}_{2}$ by the potential energy surface in the non-BO Hamiltonian, the problem was reduced to a one-pseudo-particle 3-D problem. It was realized that only when a basis set of Gaussians multiplied by a quite extended range of powers of the inter-nuclear distance, $r$, was used $\left(r^{m_{i}} \mathrm{e}^{-b_{i} r_{2}}\right)$, were the results obtained by the non-BO method very similar to those reported by Kolos and Wolniewicz. In one of the calculations a single Gaussian function was used to expand the ground state wave function, $\Psi_{0}$. This optimized wave function was:

$$
\Psi_{0}=N r^{17} \mathrm{e}^{-4.3496 r^{2}}
$$

This result shows how high the $r$ powers have to be to describe the vibrational wave function using only Gaussians located at the center of the coordinate system. This is not an unexpected result because it reflects the fact that most of the Gaussians in the basis set should have maxima located at $r$ close to the equilibrium $\mathrm{H}_{2}$ inter-nuclear distance. This can only be achieved with one-center Gaussians if the function is multiplied by a high power of $r$. The higher the power of the $r^{m}$ factor of the Gaussian the farther away its maximum is shifted from the center of the coordinate system.

The realization that in order to describe the relative motion of the two nuclei (i.e., the inter-nuclear correlation effects) in the non-BO wave function of a diatomic system one needs to use Gaussians multiplied by powers of the inter-nuclear distance convinced us that the following basis should be used be used in the non-BO calculations for diatomic molecules:

$$
\phi_{k}=r_{1}^{m_{k}} \exp \left[-\boldsymbol{r}^{\prime}\left(\boldsymbol{L}_{k} \boldsymbol{L}_{\boldsymbol{k}}^{\prime} \otimes \boldsymbol{I}_{3}\right) \boldsymbol{r}\right],
$$

where only even $m_{k}$ powers are used.

Using the basis set in eqn. (37) we performed calculations on several diatomic systems $\mathrm{s}^{5,752,53}$ including $\mathrm{H}_{2}$. It is important to note here that our method is not restricted to systems with two electrons. Since we have used the variational method where the calculated energy is an upper bound to the exact energy the quality of the results can be judged by how low energy value is obtained. As an example of what accuracy can be achieved with our method, we compare in Table 2 our results for $\mathrm{H}_{2}$ with the best results obtained by others. Our best most recent upper bound of $-1.1640250300 E_{\mathrm{h}}$, which is an improvement on our previous result of $-1.1640250232 E_{\mathrm{h}}$, is noticeably better that any of the results obtained by others. This gave us confidence that the explicitly correlated basis set we have used correctly describes the coupled electron-nuclear motion in the diatomic systems.

In the most recent work ${ }^{52}$ the non- $\mathrm{BO} \mathrm{H}_{2}$ calculations were extended to excited states with the total angular momentum equal to zero. In the conventional approaches these states 
Table 2 Energy expectation values $/ E_{\mathrm{h}}$ for the non-adiabatic ground state of the $\mathrm{H}_{2}$ molecule

\begin{tabular}{lc}
\hline-1.1640250300 & Ref. $52\left(m_{p}=1836.1526675 m_{e}\right)$ variational, \\
& 3000 basis functions, partial optimization. \\
-1.1640250232 & Ref. $5\left(m_{p}=1836.152693 m_{e}\right)$ variational, \\
& 512 basis functions, full optimization. \\
-1.164025018 & Ref. $76\left(m_{p}=1836.1527 m_{e}\right)$ high accuracy \\
& adiabatic and non-adiabatic corrections, \\
& 883 basis functions. \\
-1.16402413 & Ref. $77\left(m_{p}=1836.15 m_{e}\right)$ variational, \\
-1.1640239 & 1070 basis functions. \\
& Ref. $78\left(m_{p}\right.$ not given) quantum \\
& Monte Carlo. \\
\hline
\end{tabular}

are described as vibrational excitations, though in the non-BO calculations they correspond to simultaneous motion of the nuclei and electrons coupled to form states with zero total angular momentum. While for the lower lying states the motion of the nuclei and the motion of the electrons can be treated separately as is done in the Born-Oppenheimer calculations, for the higher states near the dissociation limit the coupling of the two motions becomes more significant. The excited state calculations rendered energies of all the vibrational states of $\mathrm{H}_{2}$ in very close agreement with the experimental data showing that the Gaussians multiplied by powers of the inter-nuclear distance are capable of describing the complicated nodal structure of the non-BO wave functions of excited states. However, to achieve accuracy similar to that obtained for the ground state the range of powers of the pre-exponential $r_{1}^{m_{k}}$ factor had be extended to 250. As may be expected, for the same number of basis functions, excited states are described less accurately than the ground state since the former have a more complicated nodal structure.

\section{Basis sets for non-Born-Oppenheimer calculations on molecules with more than two nuclei}

As shown the nucleus-nucleus correlation effects in the diatomic systems in the non-BO calculations can be effectively described by including pre-exponential powers of the internuclear distance in the one-center Gaussians. This result can be generalized to systems with more than two nuclei and an arbitrary number of electrons. This is the next step in the development of the non-BO method that has been carried out in our research group. In this development the Gaussian basis functions for triatomic systems such as $\mathrm{H}_{3}{ }^{+}, \mathrm{H}_{3}$, for states with zero total angular momentum have the following form:

$$
\phi_{k}=r_{1}^{2 m_{k}} r_{2}^{2 l_{k}} r_{12}^{2 n_{k}} \exp \left[-\boldsymbol{r}^{\prime}\left(\boldsymbol{A}_{k} \otimes \boldsymbol{I}_{3}\right) \boldsymbol{r}\right] .
$$

This basis function includes powers of distances between all pairs of the nuclei forming the system. This is necessary to describe the physical nature of the inter-nuclear correlation and to describe the nodes in the wave functions corresponding to vibrational excited states. As with the diatomic basis sets, here we only use even powers of the distances. The effect this exclusion of odd powers may have on the ground state energy of the system has been tested and found to be negligible.

In the general case with $N$ nuclei and $n \sigma$-electrons the basis basis functions should have the following form:

$$
\phi_{k}=\left(\prod_{i<j}^{N} r_{i j}^{2 m_{k i j}}\right) \exp \left[-\boldsymbol{r}^{\prime}\left(\boldsymbol{A}_{k} \otimes \boldsymbol{I}_{3}\right) \boldsymbol{r}\right]
$$

Here again the inter-nuclear distance of each pair of the nuclei is raised to a power $\left(2 m_{k i j}\right)$ and included as a pre-exponential multiplier. Derivation of the Hamiltonian integrals over these basis functions and, subsequently, their derivatives with respect to the non-linear parameters will be carried out in the next stage of our non-BO work.

As with the diatomic systems, the non-BO calculations of polyatomics will be implemented on parallel computer systems using the MPI message passing protocol. The diatomic calculations with larger basis sets show that due to separability of the integral and gradient calculations for each pair of the basis functions the algorithm is very parallel and runs with almost $100 \%$ efficiency on computer clusters with distributed operational memory (e.g. Beowulf clusters).

The most interesting future applications of the method will concern non-BO calculations of the vibrational spectrum of the $\mathrm{H}_{3} / \mathrm{H}_{3}{ }^{+} / \mathrm{H}_{3}{ }^{-}$systems and their isotopomers. As discovered in conventional $\mathrm{BO}$ calculations, there is a conical intersection of the ground and the first excited electronic state in $\mathrm{H}_{3}{ }^{+}$. Direct non-BO calculations of the vibrational manifold of this system will allow for the first time a rigorous, variational, non-adiabatic calculated description of this phenomenon.

Finally we make a comment about calculating states with the total angular momentum of electrons and nuclei not equal to zero. In calculations of rotationally excited states the nonadiabatic wave functions will be expanded as symmetry projected linear combinations of the explicitly correlated $\phi_{k}$ multiplied by an angular term, $Y_{L M}^{k}$ :

$$
\Psi_{L M \Gamma}=\mathscr{P}_{\Gamma} \sum_{k} c_{k} Y_{L M}^{k} \phi_{k}
$$

Here $\phi_{k}$ are the explicitly correlated $n$-body Gaussians given in eqn. (21), $\mathscr{P}_{\Gamma}$ is an appropriate permutational symmetry projection operator for the desired state, $\Gamma$. $Y_{L M}^{k}$ is a vectorcoupled product of solid harmonics ${ }^{54}$ given by the ClebschGordan expansion,

$$
Y_{L M}^{k}=\sum_{\left\{l_{j}, m_{j}\right\}, m_{1}+\cdots+m_{n}=M}\left\langle L M ; k \mid l_{1} m_{1} \cdots l_{n} m_{n}\right\rangle \prod_{j}^{n} \mathscr{Y}_{l_{j} m_{j}},
$$

and the solid harmonics are given by:

$$
\begin{aligned}
\mathscr{Y}_{l m}\left(\boldsymbol{r}_{j}\right)= & {\left[\frac{2 l+1}{4 \pi}(l+m) !(l-m) !\right]^{1 / 2} } \\
& \times \sum_{p} \frac{\left(-x_{j}-i y_{j}\right)^{p+m}\left(x_{j}-i y_{j}\right)^{p} z_{j}^{l-2 p-m}}{2^{2 p+m}(p+m) ! p !(l-m-2 p) !} .
\end{aligned}
$$

The $\mathscr{Y}_{l m}\left(\boldsymbol{r}_{j}\right)$ are single-particle angular momentum eigenfunctions in relative coordinates which transform the same as spherical harmonics, i.e. have the same eigenvalues. Since the $\phi_{k}$ are angular momentum eigenfunctions with zero total angular momentum, the product with $Y_{L M}^{k}$ can be used in principle to obtain any desired angular momentum eigen-state. Note the $k$-dependence of $Y_{L M}^{k}$; this is included since there are many ways to couple the individual angular momenta, $l_{j}$, to achieve the desired total angular momentum $L$ and it may be necessary to include several sets of the $l_{j}$ 's in order to obtain a realistic description of the wave function. Varga and Suzuki ${ }^{55,56}$ have recently proposed representing the angular dependence of the wave function using a single solid harmonic whose argument contains additional variational parameters, $\boldsymbol{u}=$ $\left(\boldsymbol{U}_{1}, \boldsymbol{U}_{2}, \cdots, \boldsymbol{U}_{n}\right)$ :

$$
\Psi_{L M T}=\mathscr{P}_{\Gamma} Y_{L M}(\boldsymbol{v}) \sum_{k} c_{k} \phi_{k}, \quad \text { with } \quad \boldsymbol{v}=\sum_{i=1}^{n} u_{i} \boldsymbol{r}_{i} .
$$

There appears to be several advantages in doing this and we are investigating the possibility of using this approach in our full $N$-body implementation. On the other hand the strict separation of the angular and "radial" variables in eqn. (40) 
allows separate consideration of the electronic-vibrational states with different total angular momentum quantum number, $L$. Making the angular terms in eqn. (40) only dependent on Euler angles and not on $r_{i j}$ or any other set of rotationally invariant variables, could lead to a more effective way to describe the rotational properties of the wave function. The magnitude of the Coriolis coupling for the particular $L$-state will determine whether the most general form, eqn. (40), or the more simplified form, eqn. (43), of the total wave function should be used.

\section{Non-Born-Oppenheimer calculations of molecules in external static electric fields}

When an electric field is applied the molecular Hamiltonian no longer commutes with the angular momentum $\hat{J}^{2}$ (here we use $J=R+L$ ), but, if the field is applied along the $z$-axis, it does commute with $\hat{J}_{z}$. In this case the wave function is no longer spherically symmetric because the electric field polarizes both the electronic and the nuclear components of the wave function. The basis functions used in the calculation have to be able to describe this cylindrical deformation of the wave function.

The wave function is thus expanded in a basis of floating spherical explicitly correlated Gaussians (FSECG). When the functional centers of these basis functions are allowed to vary, this basis in principle provides a complete set for the expansion (see ref. 9 or ref. 57 for a discussion of other types of basis sets which may be used). The FSECG basis function for $n$ particles is:

$$
\phi_{k}(r)=\exp \left\{-\left(\boldsymbol{r}-\boldsymbol{s}_{k}\right)^{\prime}\left[\boldsymbol{L}_{k} \boldsymbol{L}_{k}^{\prime} \otimes \boldsymbol{I}_{3}\right]\left(\boldsymbol{r}-\boldsymbol{s}_{k}\right)\right\} .
$$

The spin-free spatial wave function has the form:

$$
\Psi=\sum_{k=1}^{m} c_{k} \hat{\mathscr{P}} \phi_{k},
$$

where $m$ is the size of the basis. $\hat{\mathscr{P}}$ is the direct product of the Young operators for all of the sets of identical particles in the system of interest.

We calculate the dipole moment and the polarizabilities from total energy values determined for the ground states for different electric field strengths. The energy values are obtained as variationally optimized expectation values of the Hamiltonian operator. The wave function in eqn. (45), is optimized with respect to the parameters $\boldsymbol{L}_{k}, \boldsymbol{s}_{k}$, and $\boldsymbol{c}_{k}$. This leads to $\frac{1}{2} n(n+1)+3 n+1$ variational parameters per basis function. The analytical optimization of linear and non-linear parameters of the basis functions allows use of smaller basis set expansions than are used when basis functions are generated randomly. ${ }^{57}$ We begin with an initial guess for the wave function and then use analytical gradients in a truncated Newtontype algorithm to find the lowest value for the energy. The $i$ th order electrical property is calculated numerically from the definition:

$$
P_{i}=-\frac{\partial^{i} E}{\partial \varepsilon_{z}^{i}} .
$$

In the nonadiabatic approach, the ground state of any molecule is spherically symmetric. In principle this leads to values of exactly zero for any odd-ordered properties based on the above definitions. This is in fact correct for any unperturbed ground state molecule. In the absence of any electric field, the wave function has full spherical symmetry and commutes with the $\hat{J}^{2}$ operator. Thus by definition it does not have a dipole moment, or first hyperpolarizability, etc.

The properties are calculated by fitting a polynomial to a series of energies as a function of electric field strength. It should be obvious that in a nonadiabatic calculation, applying a negative field should be equivalent to applying a positive field since the molecule, not being fixed in space, is free to rotate. Thus a fit of the energy for both positive and negative field strengths would still give a dipole moment of zero, consistant with the above explanation. This can be overcome by using only positive field strengths in the polynomial fit. This trick allows a high accuracy calculation of dipole moments, as shown in the previous work..$^{8,10}$

Since the basis functions used here are eigenfunctions of $\hat{J}_{z}$, but not $\hat{J}^{2}$, they are of correct symmetry only for the non-zerofield calculations. In order to maintain equal representation of all the points in the fit, though, they are used for the zero field point as well. This should introduce only mild contamination by excited rotational states and has not been a practical problem in the past.

\section{Examples of non-Born-Oppenheimer electrical property calculations}

The smallest systems investigated by the current method have been $\mathrm{H}_{2}$ and its isotopomer series. For these calculations basis sets of 16 terms were used for the homonuclear (HON) species, and 32 terms for the heteronuclear (HEN) species. These relative sizes were used in order to have results of comparable quality since the HEN wave functions include projection onto irreducible representations of the symmetric group $S_{2}^{\text {e which }}$ takes into account electron permutation (two terms) while the HON wave functions include projection onto irreducible representations of $S_{2}^{\mathrm{e}} \otimes S_{2}^{\mathrm{N}}$ which takes into account electronic and nuclear exchange (four terms). The wave functions were fully optimized for each isotopomer at seven field strengths: $0,0.0004,0.0008,0.0016,0.0032,0.0064$, and $0.0128 \mathrm{au}$. The energies obtained at each field strength for each isotopomer were plotted and fitted to a fourth order polynomial in field strength and the properties were extracted from the coefficients of the fit.

While the basis sets used in these calculations were small and the zero field energies were not converged with respect to basis set size, the calculated properties were of good quality. As shown in Table 3, the dipole moment of HD, 0.000327 au is in excellent agreement with other theoretical values (see previous work; ${ }^{10}$ average of previous theoretical values is $0.000327 \mathrm{au}$ ) and close to the experimentally measured value of $0.000345 \mathrm{au}^{12}$ The relative values of the other dipole moments may be predicted based on the relative electronegativities of $\mathrm{H}, \mathrm{D}$, and $\mathrm{T}$, and these predicted values agree with the calculated values of Table 3. The values for the higher order properties also agree with previous values (see previous work $\left.{ }^{10}\right)$. The dipole moments and first hyperpolarizabilities for the HON should be identically zero, and the values shown in the table should give an indication of the numerical error present in the fitting procedure. As may be expected, the higher

Table 3 Non-BO energies (at zero-field), dipole moments $(\mu)$, polarizabilities $(\alpha)$, hyperpolarizabilities $(\beta)$ and second hyperpolarizabilities $(\gamma)$, for the $\mathrm{H}_{2}$ isotopomer series. All values are in atomic units ${ }^{10}$

\begin{tabular}{lllllr}
\hline & $\langle H\rangle$ & $\mu$ & $\alpha$ & $\beta$ & $\gamma$ \\
\hline $\mathrm{H}_{2}$ & -1.153736345 & $1.00 \times 10^{-8}$ & 6.74 & $(0.0360)$ & 1062 \\
$\mathrm{HD}$ & -1.156234289 & $3.27 \times 10^{-4}$ & 6.67 & $(0.0306)$ & 1038 \\
$\mathrm{HT}$ & -1.157152576 & $4.37 \times 10^{-4}$ & 6.65 & $(0.0186)$ & 1028 \\
$\mathrm{D}_{2}$ & -1.159178760 & $9.00 \times 10^{-9}$ & 6.59 & $(0.0360)$ & 1009 \\
$\mathrm{DT}$ & -1.160312051 & $1.09 \times 10^{-4}$ & 6.56 & $(0.0312)$ & 999 \\
$\mathrm{~T}_{2}$ & -1.161561149 & $9.00 \times 10^{-9}$ & 6.52 & $(0.0360)$ & 989 \\
Experiment, & & $3.45 \times 10^{-4}$ & & & \\
$\quad$ & & & & & \\
\hline
\end{tabular}


Table 4 Energies, virial coefficients $(\eta)$, and dipole moments $(\mu)$, for non-BO LiH/D for various expansion lengths $(m)$. All values are in atomic units 8

\begin{tabular}{llll}
\hline$m$ & $\langle H\rangle$ & $\eta$ & $\mu$ \\
\hline $\mathrm{LiH}$ & & & \\
24 & -8.0423294 & 1.000000 & 2.4047 \\
64 & -8.0592988 & 1.000000 & 2.3394 \\
104 & -8.0619627 & 1.000000 & 2.3261 \\
144 & -8.0629324 & 0.999999 & 2.3149 \\
244 & -8.0636331 & 0.999999 & 2.3140 \\
Experimental & & & $2.314 \pm 0.001$ \\
& & & $($ ref. 11) \\
LiD & & & \\
244 & -8.0650331 & 1.000000 & 2.3088 \\
Experimental & & & $2.309 \pm 0.001$ (ref. 11) \\
\hline
\end{tabular}

order properties have larger errors. Also, the first hyperpolarizabilities of the HEN are too small and too near the noise level to be reliable.

$\mathrm{LiH}$ is the largest system studied so far with this method. For LiH a series of basis sets was used: $24,64,104,144$, and 244 terms; each basis was projected onto the symmetric group $S_{4}$ which has 24 terms. As may be seen in Table 4, each basis set is well converged (as shown by the value of the virial coefficient), and the energy is converging well with the series, though it is not as low as the current nonadiabatic upper bound for the $\mathrm{LiH}$ ground state. ${ }^{7}$ The expectation value for the inter-nuclear distance for the largest basis set used, $3.063 \mathrm{au}$, is quite close to that found in the other work, ${ }^{7} 3.061 \mathrm{au}$. The energy and wave function for each basis was calculated at several field strengths: $0,0.0016,0.0032$, and 0.0048 au. These energies were fit to a third order polynomial and the properties were extracted from this fit.

More recently, these same calculations were done in a slightly different way. ${ }^{31}$ In this attempt, more field strengths were used $(0,0.0004,0.0008,0.0016,0.0032$, and 0.0064) and a fourth order polynomial was used, although the calculations were only carried out for the three smallest basis sets. The dipole moments found in this approach were identical to those found in the work described above. The polarizabilities did change slightly, as would be expected. This increase in the polarizabilities is at maximum slightly less than $1 \%$ and the values presented in Table 4 are sufficient for the discussion here. In any case, though, the converged values for the polarizability are in good agreement with previously calculated electronic + nuclear values. The converged values of the dipole moments for the largest basis sets for $\mathrm{LiH}$ and $\mathrm{LiD}$ $(2.3140,2.3088 \mathrm{au})$ agree very well with experiment (2.3140, $2.3090 \mathrm{au})^{11}$

\section{Non-Born-Oppenheimer calculations on larger molecular systems}

The implementation of the basis set consisting of explicitly correlated Gaussians with shifted centers, $\phi_{k}=$ $\exp \left[-(\boldsymbol{r}-\mathbf{s})^{\prime}\left(\boldsymbol{A}_{k} \otimes \boldsymbol{I}_{3}\right)(\boldsymbol{r}-\mathbf{s})\right]$, allows the calculation of wave functions for systems with more than two nuclei with the non-Born-Oppenheimer method. Though these basis functions are not eigenfunctions of the square of the total angular momentum operator as are the functions without shifts, $\phi_{k}=\exp \left[-\boldsymbol{r}^{\prime}\left(\boldsymbol{A}_{k} \otimes \boldsymbol{I}_{3}\right) \boldsymbol{r}\right]$, they can still be used to obtain very good non-adiabatic wave functions since they form a complete set and, in principle, can describe any non-BO bound state of a molecular system. However, since they are not eigenfunctions of the total angular momentum, it is difficult to use them to calculate excited states in a way that would allow separation of vibrational-electronic and rotational excitations. Such separation automatically occurs when one uses basis functions that are angular momentum eigenfunctions. In order to generate a wave function corresponding to the specific rotational excitation in the basis of shifted correlated Gaussians, one would need to project out from the wave function the contributions from angular momenta different from the angular momentum of the calculated state. This may not be a practical way to proceed, especially if very high accuracy is required.

Thus, these basis functions may practically be used to calculate only ground states of polyatomic systems. In some preliminary calculations in this area we are considering the $\mathrm{H}_{3}{ }^{+} /$ $\mathrm{H}_{3}$ system and its isotopomers. The goal of the calculations is to determine the isotopic effect on the adiabatic ionization potential of $\mathrm{H}_{3}$. The results will be published in a forthcoming paper. An interesting aspect of these types of calculations is the way a good starting guess of the non-BO wave function may be generated. First, a conventional variational BO calculation for the system using a basis set of correlated Gaussians with shifted centers is performed. For this the method developed recently $^{58}$ that allows simultaneous optimization of the molecular geometry and the non-linear parameters (i.e. exponents and centers) of the basis functions is used. Next the converged BO wave function is multiplied by Gaussians dependent on the coordinates of the nuclei. These Gaussians are centered at the equilibrium positions of the nuclei that were obtained in the BO calculation (with the reference nucleus placed at the center of the coordinate system).

The non-BO method that we have been developing is general and can be applied, in principle, to systems with any number of nuclei and electrons. However, the complexity of the calculations increases very rapidly ( $n$ factorial) with the increase of the number of identical particles. Thus it is desireable to develop a hybrid method which describes some of the nuclei within the BO approximation and treats some of the light nuclei (protons) as quantum particles. Recently some interesting developments have been reported where such an equivalent representation has been proposed. In a series of papers Tachikawa, Nakai and coworkers ${ }^{59-62}$ introduced a variational molecular orbital theory for constructing a wave functions dependent on both electrons and nuclei coordinates. In their work the wave function was a product of single Slater determinants for the electrons and for the protons (and any other fermion particles in the system), and orbitals representing the boson particles. The method was a straight forward extension of the Hartree-Fock theory for electrons. The heavier nuclei in their calculations were represented by point charges and not included in the wave function. The calculations were done using cartesian Gaussian basis functions. Their more recent work extended the method to configuration-interaction (CI) type wave functions with single excitations to describe excited states. In a parallel development Hammes-Schiffer and coworkers $^{3}$ presented a similar scheme termed the nuclearelectronic orbital (NEO) method where the CI wave function representing nuclei and electrons included singly and doubly excited determinants and in their scheme they optimized both configurational and orbital linear expansion parameters (MCSCF-type wave function).

The correlated Gaussians with shifted centers form a very convenient basis for a hybrid $\mathrm{BO} /$ non-BO approach. Below we describe the major points of a hybrid approach being considered in our lab. Let us take a molecular system consisting of light nuclei (protons), heavy nuclei (carbon, nitrogen, oxygen, etc.) and electrons. For the sake of simplicity we assume that the light nuclei are all protons. An extension to include other light nuclei, such as deuterium or lithium nuclei, is straight forward. At first we make an approximation that the heavy nuclei are treated using the Born-Oppenheimer approximation and the wave function only parametrically depends on their 
coordinates. In some cases we may also choose to treat some not essential protons that way. Thus the internal Hamiltonian and the wave function explicitly depends only on the coordinates of the "active" protons and all the electrons in the studied system. In the Hamiltonian additional terms representing the interaction of the active particles with the non-active particles appear:

$$
\begin{aligned}
\hat{H}= & -\frac{1}{2}\left(\left(\sum_{i}^{n} \frac{1}{m_{i}} \nabla_{i}^{2}+\sum_{i \neq j}^{n} \frac{1}{M_{1}} \nabla_{i} \cdot \nabla_{j}\right)\right. \\
& +\sum_{i=1}^{n} \frac{q_{0} q_{i}}{r_{i}}+\sum_{i<j}^{n} \frac{q_{i} q_{j}}{r_{i j}}+\sum_{i}^{n} \sum_{k}^{K} \frac{q_{i} q_{k}}{r_{i k}},
\end{aligned}
$$

where $q_{i}$ and $q_{k}$ are charges of the active particles and the nonactive nuclei, respectively, and the $r_{i k}$ is the distance between them.

The variational method will be used to determine ground and excited-state energies and the corresponding wave functions. In the variational calculations we will optimize the linear and non-linear parameters of the orbital part and the correlated part of the wave function, as well as the positions of the "inactive" nuclei (i.e. heavy nuclei and inactive light nuclei).

The development of a hybrid method that combines the BO description of heavier nuclei with quantum-mechanical nonBO description of light nuclei and electrons evolves from the realization that at present the application of our non-BO approach to larger molecular systems is not practical due to computational limitations. However, our previous studies of the inter- and intra-molecular proton transfer reactions, ${ }^{63-72}$ as well as the studies of others (notably, the high accuracy quantum scattering work on multiple potential energy surfaces by Schatz, et $a l .{ }^{73}$ ), have indicated that in the theoretical characterization of those reactions it is important to describe quantum tunneling and non-adiabatic effects. The challenging task of developing an accurate non-BO method to study intra- and inter-molecular proton transfer reactions in one of the aims of this work. Since an important aspect of these reactions is the quantum-mechanical behavior of the protons and, in some situations, coupling of the proton and electron motions (non-adiabatic behavior), the new method has to incorporate these effects. This will be done by merging of the fully nonadiabatic method developed in our group for small molecular systems that utilizes explicitly correlated Gaussian functions, with the conventional orbital-based BO approach. After separating the center-of-mass motion from the Hamiltonian, as described above, the hybrid wave function which will be used will have the following form:

$$
\begin{aligned}
\Psi\left(\boldsymbol{r}_{1},\right. & \left.\boldsymbol{r}_{2}, \ldots, \boldsymbol{r}_{n}, \boldsymbol{R}_{1}, \boldsymbol{R}_{2}, \ldots, \boldsymbol{R}_{M} ; \boldsymbol{Q}_{1}, \boldsymbol{Q}_{2}, \ldots, \boldsymbol{Q}_{K}\right) \\
= & \mathscr{A}\left(\boldsymbol{r}_{1}, \boldsymbol{r}_{2}, \ldots, \boldsymbol{r}_{n} ; \boldsymbol{R}_{1}, \boldsymbol{R}_{2}, \ldots, \boldsymbol{R}_{M}\right) \\
& \times\left[\Phi\left(\boldsymbol{r}_{1}, \boldsymbol{r}_{2}, \ldots, \boldsymbol{r}_{n}\right) \psi\left(\boldsymbol{R}_{1} ; \boldsymbol{Q}_{1}, \boldsymbol{Q}_{2}, \ldots, \boldsymbol{Q}_{K}\right)\right. \\
& \times \psi_{2}^{\mathrm{e}}\left(\boldsymbol{R}_{3} ; \boldsymbol{Q}_{1}, \boldsymbol{Q}_{2}, \ldots, \boldsymbol{Q}_{K}\right) \cdots \psi_{N}^{\mathrm{e}} \\
& \left.\times\left(\boldsymbol{R}_{M} ; \boldsymbol{Q}_{1}, \boldsymbol{Q}_{2}, \ldots, \boldsymbol{Q}_{K}\right)\right],
\end{aligned}
$$

where the $\boldsymbol{R}_{1}, \boldsymbol{R}_{2}, \ldots, \boldsymbol{R}_{n}$ coordinates refer to the active light nuclei and their electrons, the $\boldsymbol{R}_{1}, \boldsymbol{R}_{2}, \ldots, \boldsymbol{R}_{M}$ coordinates refer to the the electrons that are described with the orbital basis functions of Cartesian Gaussian orbitals centered on inactive (stationary) atoms, the $\mathbf{Q}_{1}, \mathbf{Q}_{2}, \ldots, \mathbf{Q}_{K}$ coordinates are the coordinates the nuclei of the stationary atoms, and the $\mathscr{A}$ operator imposes the appropriate permutational symmetry of the electrons and the identical light nuclei in the wave function. In the wave function, eqn. (48), we assume that the electronic orbitals, $\psi_{i}^{\mathrm{e}}$, and the nuclear orbitals, $\psi_{i}^{\mathrm{n}}$, form two orthogonal sets of functions. As in our previous non-BO calculations, we will use the variational method to determine the energies and the wave functions for the ground and excited states of the system. In the variational optimization of the wave function, eqn. (48), we will optimize both linear and non-linear parameters of $\Phi\left(\boldsymbol{R}_{1}, \boldsymbol{R}_{2}, \ldots, \boldsymbol{R}_{n}\right)$, the linear parameters of the electronic and nuclear orbitals, $\psi_{i}^{\mathrm{e}}$ and $\psi_{i}^{\mathrm{n}}$, as well as the positions of the inactive nuclei. This will be accomplished using algorithms based on analytical gradients that we have developed for the $\mathrm{BO}$ and non-BO calculations.

The fixed positions of the "inactive" nuclei, even if they are fully optimized for each considered state, could be the major source of error if the wave function in eqn. (48) is used in the calculations. An alternative, which will be considered, includes quantum behavior of the "inactive" nuclei with the use of the harmonic approximation. The wave function that will account for these effects will have the following form:

$$
\begin{aligned}
\Psi\left(\boldsymbol{r}_{1},\right. & \left.\boldsymbol{r}_{2}, \ldots, \boldsymbol{r}_{n}, \boldsymbol{R}_{1}, \boldsymbol{R}_{2}, \ldots, \boldsymbol{R}_{M}, \boldsymbol{Q}_{1}, \boldsymbol{Q}_{2}, \ldots, \boldsymbol{Q}_{K}\right) \\
= & \mathscr{A}\left(\boldsymbol{r}_{1}, \boldsymbol{r}_{2}, \ldots, \boldsymbol{r}_{n}, \boldsymbol{R}_{1}, \boldsymbol{R}_{2}, \ldots, \boldsymbol{R}_{M}\right) \\
& \times\left[\Phi\left(\boldsymbol{r}_{1}, \boldsymbol{r}_{2}, \ldots, \boldsymbol{r}_{n}\right) \psi_{1}^{\mathrm{e}}\left(\boldsymbol{R}_{1} ;\left\langle\boldsymbol{Q}_{1}\right\rangle,\left\langle\boldsymbol{Q}_{2}\right\rangle, \ldots,\left\langle\boldsymbol{Q}_{K}\right\rangle\right)\right. \\
& \times \psi_{2}^{\mathrm{e}}\left(\boldsymbol{R}_{3} ;\left\langle\boldsymbol{Q}_{1}\right\rangle,\left\langle\boldsymbol{Q}_{2}\right\rangle, \ldots,\left\langle\boldsymbol{Q}_{K}\right\rangle\right) \cdots \psi_{N}^{\mathrm{e}}\left(\mathbf{R}_{M}\right. \\
& \left.\left.\times\left\langle\boldsymbol{Q}_{1}\right\rangle,\left\langle\boldsymbol{Q}_{2}\right\rangle, \ldots,\left\langle\boldsymbol{Q}_{K}\right\rangle\right)\right] \psi_{1}^{\mathrm{n}}\left(\boldsymbol{Q}_{1}\right) \psi_{2}^{\mathrm{n}}\left(\boldsymbol{Q}_{2}\right) \cdots \psi_{K}^{\mathrm{n}}\left(\boldsymbol{Q}_{K}\right)
\end{aligned}
$$

where the $\psi_{i}^{\mathrm{n}}\left(\boldsymbol{P}_{i}\right)$ functions describe the ground vibrational states of the inactive nuclei in the harmonic approximation. These functions are linear combinations of simple s-type spherical Gaussians with floating centers. The orbitals of the inactive nuclei, $\psi_{1}^{\mathrm{e}}\left(\boldsymbol{R}_{1} ;\left\langle\boldsymbol{Q}_{1}\right\rangle,\left\langle\boldsymbol{Q}_{2}\right\rangle, \ldots,\left\langle\boldsymbol{Q}_{K}\right\rangle\right)$, still show dependence on the inactive-nuclei positions, though in this case the coordinates describing these positions are variables and not parameters in the wave function in eqn. (48). However, with the notation $\left\langle\boldsymbol{P}_{i}\right\rangle$ we indicate that the electronic orbitals are not centered at the moving inactive nuclei, but rather at some average nuclei positions that are subject to the variational optimization as parameters of the wave function.

One can notice that in the above wave function the inactive nuclei and their electrons are described with the use of oneparticle functions and they are not explicitly correlated in the way the active nuclei and their electrons are correlated. This approximation should hold well for heavier nuclei since their displacements from the equilibrium positions in the zero-point vibrations should be small in comparison to the displacements of the light active nuclei. The one-particle approximation is made to reduce the computational costs of the calculations since it allows use of mutually orthogonal electronic orbitals, $\psi_{i}^{\mathrm{e}}\left(\boldsymbol{R}_{i} ;\left\langle\boldsymbol{Q}_{1}\right\rangle,\left\langle\boldsymbol{Q}_{2}\right\rangle, \ldots,\left\langle\boldsymbol{Q}_{K}\right\rangle\right)$, and orthogonal nuclear orbitals, $\psi_{1}^{\mathrm{n}}\left(\boldsymbol{Q}_{1}\right)$, in the calculation.

Another source of error in the wave function in eqn. (48) is the lack of correlation of the electrons of the inactive nuclei, as well as the correlation between the electron of the inactive nuclei and the active electrons. This missing correlation can be described by using a configuration-interaction expansion of the electronic part of the wave function with single- and double-excitation operators generated by the excitation operators, $E_{i}^{a}, E_{i j}^{a b}$, in the following way:

$$
\begin{aligned}
\Psi & \left(\boldsymbol{r}_{1}, \boldsymbol{r}_{2}, \ldots, \boldsymbol{r}_{n}, \boldsymbol{R}_{1}, \boldsymbol{R}_{2}, \ldots, \boldsymbol{R}_{M}, \boldsymbol{Q}_{1}, \boldsymbol{Q}_{2}, \ldots, \boldsymbol{Q}_{K}\right) \\
& \times \mathscr{A}\left(\boldsymbol{r}_{1}, \boldsymbol{r}_{2}, \ldots, \boldsymbol{r}_{n}, \boldsymbol{R}_{1}, \boldsymbol{R}_{2}, \ldots, \boldsymbol{R}_{M}\right) \\
& \times\left[\Phi\left(\boldsymbol{r}_{1}, \boldsymbol{r}_{2}, \ldots, \boldsymbol{r}_{n}\right)\left(1+\sum_{i, a} c_{i}^{a} E_{i}^{a}+\sum_{i, j, a, b} c_{i j}^{a b} E_{i j}^{a b}\right) \psi_{1}^{\mathrm{e}}\right. \\
& \times\left(\boldsymbol{R}_{1} ;\left\langle\boldsymbol{Q}_{1}\right\rangle,\left\langle\boldsymbol{Q}_{2}\right\rangle, \ldots,\left\langle\boldsymbol{Q}_{K}\right\rangle\right) \psi_{2}^{\mathrm{e}}\left(\boldsymbol{R}_{3} ;\left\langle\boldsymbol{Q}_{1}\right\rangle,\left\langle\boldsymbol{Q}_{2}\right\rangle, \ldots,\right. \\
& \left.\left.\times\left\langle\boldsymbol{Q}_{K}\right\rangle\right) \cdots \psi_{N}^{\mathrm{e}}\left(\boldsymbol{R}_{M} ;\left\langle\boldsymbol{Q}_{1}\right\rangle,\left\langle\boldsymbol{Q}_{2}\right\rangle, \ldots,\left\langle\boldsymbol{Q}_{K}\right\rangle\right)\right] \\
& \times \psi_{1}^{\mathrm{n}}\left(\boldsymbol{Q}_{1}\right) \psi_{2}^{\mathrm{n}}\left(\boldsymbol{Q}_{2}\right) \cdots \psi_{K}^{\mathrm{n}}\left(\boldsymbol{Q}_{K}\right) .
\end{aligned}
$$

The excitation operators are defined using orthonormal orbitals obtained from a variational Hartree-Fock-like calculation of the wave function in eqn. (49). The wave function in eqn. (50) can be further generalized by making the expansion coefficients of the component of the wave function that describes the 
active nuclei and their electrons dependent on the excitations of the other electrons:

$$
\begin{aligned}
\Psi\left(\boldsymbol{r}_{1},\right. & \left.\boldsymbol{r}_{2}, \ldots, \boldsymbol{r}_{n}, \boldsymbol{R}_{1}, \boldsymbol{R}_{2}, \ldots, \boldsymbol{R}_{M}, \boldsymbol{Q}_{1}, \boldsymbol{Q}_{2}, \ldots, \boldsymbol{Q}_{K}\right) \\
= & \mathscr{A}\left(\boldsymbol{r}_{1}, \boldsymbol{r}_{2}, \ldots, \boldsymbol{r}_{n}, \boldsymbol{R}_{1}, \boldsymbol{R}_{2}, \ldots, \boldsymbol{R}_{M}\right) \\
& \times\left[\left(\Phi\left(\boldsymbol{r}_{1}, \boldsymbol{r}_{2}, \ldots, \boldsymbol{r}_{n}\right)+1+\sum_{i, a} c_{i}^{a} \Phi_{i a}\left(\boldsymbol{r}_{1}, \boldsymbol{r}_{2}, \ldots, \boldsymbol{r}_{n}\right) E_{i}^{a}\right.\right. \\
& \left.+\sum_{i, j, a, b} c_{i j}^{a b} \Phi_{i j a b}\left(\boldsymbol{r}_{1}, \boldsymbol{r}_{2}, \ldots, \boldsymbol{r}_{n}\right) E_{i j}^{a b}\right) \psi_{1}^{\mathrm{e}}\left(\boldsymbol{R}_{1} ;\left\langle\boldsymbol{Q}_{1}\right\rangle,\left\langle\boldsymbol{Q}_{2}\right\rangle\right. \\
& \left.\times, \ldots,\left\langle\boldsymbol{Q}_{K}\right\rangle\right) \psi_{2}^{\mathrm{e}}\left(\boldsymbol{R}_{3} ;\left\langle\boldsymbol{Q}_{1}\right\rangle,\left\langle\boldsymbol{Q}_{2}\right\rangle, \ldots,\left\langle\boldsymbol{Q}_{K}\right\rangle\right) \cdots \psi_{N}^{\mathrm{e}} \\
& \left.\times\left(\boldsymbol{R}_{M} ;\left\langle\boldsymbol{Q}_{1}\right\rangle,\left\langle\boldsymbol{Q}_{2}\right\rangle, \ldots,\left\langle\boldsymbol{Q}_{K}\right\rangle\right)\right] \psi_{1}^{\mathrm{n}}\left(\boldsymbol{Q}_{1}\right) \psi_{2}^{\mathrm{n}}\left(\boldsymbol{Q}_{2}\right) \cdots \psi_{K}^{\mathrm{n}}\left(\boldsymbol{Q}_{K}\right) .
\end{aligned}
$$

The above sequence of the wave functions, eqns. (48-51), demonstrates that the accuracy level in this approach can be systematically improved by increasing the complexity of the wave function. The improvement can also be accomplished by increasing the number of active nuclei and the number of electrons described by the $\Phi\left(\boldsymbol{r}_{1}, \boldsymbol{r}_{2}, \ldots, \boldsymbol{r}_{n}\right)$ component in the wave function, which is expanded in terms of $n$-particle explicitly-correlated Gaussian functions that, as we have demonstrated in our previous non-BO calculations, can very accurately describe highly correlated states of multi-particle systems with Coulombic interactions.

As mentioned in our non-BO calculations with the explicitly correlated Gaussians with shifted centers, the variational optimization of the wave function uses an initial guess derived from the wave function obtained using the $\mathrm{BO}$ approximation. This approach, which will also be used in this hybrid method, starts with a conventional BO, say Hartree-Fock, geometry optimization of the system which results in a particular equilibrium geometry. This geometry is moved in the Cartesian coordinate frame in such a way that the nucleus that in the non-BO calculation is selected as the center of the internal coordinate system is positioned in the center of the frame. Next the electronic wave function obtained in the BO calculation is modified to include the $\Phi\left(\boldsymbol{r}_{1}, \boldsymbol{r}_{2}, \ldots, \boldsymbol{r}_{n}\right)$ component describing the active particles (and exclude the electrons described by this component from the $\mathrm{BO}$ wave function). The active nuclei in this component are represented by spherical Gaussian orbitals placed at their respective equilibrium positions obtained from the $\mathrm{BO}$ calculation and their electrons are described by correlated Gaussians. Such a wave function, as our calculations for small molecular systems indicate, provides an excellent starting guess to initiate the non-BO variational calculation.

\section{Summary}

In this review we described our recent work on development and implementation of a method that allows description atomic and molecular systems without assuming the BornOppenheimer approximation regarding the separability of the electronic and nuclear motions. The centerpiece of the development has been the introduction of new types of explicitly correlated basis functions in the non-BO calculations. So far we have used three types of Gaussian explicitly-correlated basis sets in our non-BO calculations: the simplest one without $r_{i j}$ pre-multipliers $\left(\phi_{k}=\exp \left[-\boldsymbol{r}^{\prime}\left(A_{k} \otimes I_{3}\right) r\right]\right)$ in atomic calculations, with pre-multipliers in the form of powers of $r_{1}$ $\left(\phi_{k}=r_{1}^{m_{k}} \exp \left[-\boldsymbol{r}^{\prime}\left(A_{k} \otimes I_{3}\right) \boldsymbol{r}\right]\right)$ in calculations for diatomic systems, and Gaussians with shifted centers $\left(\phi_{k}=\exp \right.$ $\left.\left[-(\boldsymbol{r}-\mathbf{s})^{\prime}\left(A_{k} \otimes I_{3}\right)(\boldsymbol{r}-\mathbf{s})\right]\right)$ in non-BO calculations of diatomic molecules in the static electric field. The future work will involve implementation of new basis functions for triatomic systems, as well as systems with more than three nuclei and systems with $\sigma$ and $\pi$ electrons. We will also work on implementing methods to describe rotational excited states.

The motivation for developing the non-adiabatic approach to describe the states of molecules stems from the realization that in order to reach "spectroscopic" accuracy in quantummechanical calculations (i.e., error less than $1 \mu E_{\mathrm{h}}$ ), one needs to account for the coupling between motions of electrons and nuclei and for the relativistic effects. Modern experimental techniques, such as gas-phase ion-beam spectroscopy, reach accuracy on the order of $0.001 \mathrm{~cm}^{-1}\left(5 \mathrm{n} E_{\mathrm{h}}\right){ }^{74}$ In order for molecular quantum mechanics to continue providing assistance in resolving and assigning experimental spectra and in studies of reaction dynamics, work has to continue on development of more refined theoretical methods, which account for non-adiabatic interactions. With such methods fundamental notions of molecular quantum mechanics can be explored and the basic theoretical framework of the high-resolution molecular spectroscopy can be tested.

There has been a continuing interest in theoretically describing molecular systems with a method which treats the motions of both nuclei and electrons equivalently. This type of method has to entirely depart from the PES concept. It is particularly interesting how in this type of approach the conventional notions of molecular structure and chemical bonding will be represented. In particular, the concept of chemical bonding, which at the BO level is an electronic phenomenon, is in the non-BO approach described as an effect derived from collective dynamical behavior of both electrons and nuclei.

Finally, the development of new algorithms in atomic and molecular non-BO calculations must be carried out in parallel to the development of the computer technology, particularly in advances in parallel computing. Metacomputing on a grid of distributed computing platforms connected via high-speed networks can revolutionize computational research by enabling hybrid computations that integrate multiple systems distributed over wide geographical locations. ${ }^{75}$ The non-BO method developed in our work is very well suited for implementation on parallel computational platforms. In our laboratory we use parallel "Beowulf" clusters based on commercially available PC components and connected via a fast Ethernet switch. The clusters use Unix (Linux) operational system and the software parallelism is facilitated by MPI. For our non-BO calculations we found the Beowulf clusters to be very cost-effective since the calculations can be easily distributed over a network of processors and executed with very high degree of parallelism. The nodes on the cluster do not need to share common operational memory. Utilization of massively parallel systems has given us momentum to proceed with the development of the non-BO method which we hope the computers of the future will allow to apply to larger systems more central to chemistry. Hence, while the applications presented in this review concern very small systems, the emphasis in the development we have carried out is placed on creating a general method that is applicable to molecular systems with an arbitrary number of electrons and nuclei.

\section{Acknowledgements}

This work has been supported by the National Science Foundation. M. Cafiero would like to thank the Sloan Foundation for support.

\section{References}

1 N. C. Handy and A. M. Lee, Chem. Phys. Lett., 1996, 252, 425.

2 M. Tachikawa, K. Mori, K. Suzuki and K. Iguchi, Int. J. Quantum Chem., 1998, 70, 491. 
3 S. P. Webb, T. Iordanov and S. Hammes-Schiffer, J. Chem. Phys., 2002, 117, 4106.

4 H. Nakai, K. Sodeyama and M. Hoshino, Chem. Phys. Lett., 2001, 345, 118 .

5 D. B. Kinghorn and L. Adamowicz, Phys. Rev. Lett., 1999, 83, 2541 .

6 D. B. Kinghorn and L. Adamowicz, J. Chem. Phys., 2000, 113, 1203.

7 E. Scheu, D. B. Kinghorn and L. Adamowicz, J. Chem. Phys, 2001, 114, 3393.

8 M. Cafiero and L. Adamowicz, Phys. Rev. Lett., 2002, 88, 33002.

9 M. Cafiero and L. Adamowicz, J. Chem. Phys., 2002, 116, 5557.

10 M. Cafiero and L. Adamowicz, Phys. Rev. Lett., 2002, 89, 73001.

11 L. Wharton, L. P. Gold and W. Klemperer, J. Chem. Phys., 1962, 37, 2149.

12 J. B. Nelson and G. C. Tabisz, Phys. Rev. A, 1983, 28, 2157.

13 V. I. Korobov, Phys. Rev. A, 2001, 63, 44501.

14 D. M. Bishop and S. A. Solunac, Phys. Rev. Lett., 1985, 55, 1986.

15 P. M. Kozlowski and L. Adamowicz, J. Chem. Phys., 1991, 95, 6681.

16 P. M. Kozlowski and L. Adamowicz, J. Comput. Chem., 1992, 13, 602 .

17 P. M. Kozlowski and L. Adamowicz, J. Chem. Phys., 1992, 97, 5063.

18 P. M. Kozlowski and L. Adamowicz, Phys. Rev., 1993, A48, 1903.

19 P. M. Kozlowski and L. Adamowicz, Chem. Rev., 1993, 93, 2007.

20 Z. Zhang, P. M. Kozlowski and L. Adamowicz, J. Comp. Chem., 1994, 15, 54.

21 P. M. Kozlowski and L. Adamowicz, Int. J. Quantum Chem., 1995, 55, 245

22 P. M. Kozlowski and L. Adamowicz, Int. J. Quantum Chem., $1995, \mathbf{5 5}, 367$.

23 P. M. Kozlowski and L. Adamowicz, J. Phys. Chem., 1996, 100, 6266.

24 D. W. Gilmore, P. M. Kozlowski, D. B. Kinghorn and L. Adamowicz, Int. J. Quantum Chem., 1997, 63, 991.

25 P. M. Kozlowski and L. Adamowicz, J. Chem. Phys., 1992, 96, 9013.

26 D. B. Kinghorn, Int. J. Quantum Chem., 1996, 57, 142.

27 D. B. Kinghorn and R. D. Poshusta, Phys. Rev. A, 1996, 47, 3671.

28 D. M. Bishop, Rev. Mod. Phys., 1990, 62, 343.

29 D. M. Bishop, Adv. Quantum Chem., 1994, 25, 1.

30 D. M. Bishop, Adv. Chem. Phys., 1998, 104, 1.

31 M. Cafiero, L. Adamowicz, M. Duran and J. M. Luis, J. Mol. Struct. (THEOCHEM), in press.

32 G. C. Schatz and M. A. Ratner, Quantum Mechanics in Chemistry, Dover:Mineola, NY, 2002

33 L. Adamowicz and A. J. Sadlej, J. Chem. Phys., 1977, 67, 4398.

34 L. Adamowicz and A. J. Sadlej, Chem. Phys. Lett., 1977, 48, 305.

35 L. Adamowicz, Int J. Quantum Chem., 1978, 13, 265.

36 L. Adamowicz, Acta Phys. Pol., A, 1978, 53, 471.

37 L. Adamowicz and A. J. Sadlej, J. Chem. Phys., 1978, 69, 3992.

38 L. Adamowicz and A. J. Sadlej, Acta Phys. Pol., A, 1978, 54, 73.

39 L. Adamowicz and A. J. Sadlej, Chem. Phys. Lett., 1978, 53, 377.

40 K. Szalewicz, L. Adamowicz and A. J. Sadlej, Chem. Phys. Lett., 1979, 61, 548 .

41 R. Bukowski, B. Jeziorski and K. Szalewicz, J. Chem. Phys., 1994, 100, 1366; R. Bukowski, B. Jeziorski, S. Rybak and K. Szalewicz, J. Chem. Phys., 1995, 102, 888; R. Bukowski, B. Jeziorski and K. Szalewicz, J. Chem. Phys., 1996, 104, 3306; H. L. Williams, T. Korona, R. Bukowski, B. Jeziorski and K. Szalewicz, Chem. Phys. Lett., 1996, 262, 431; T. Korona, H. L. Williams, R. Bukowski, B. Jeziorski and K. Szalewicz, J. Chem. Phys., 1997, 106, 5109, and references therein.
42 W. Cencek, J. Komasa and J. Rychlewski, Chem. Phys. Lett., $1995, \mathbf{2 4 6}, 417$

43 W. Cencek and J. Rychlewski, J. Chem. Phys., 1993, 98, 1252.

44 W. Cencek, J. Komasa and J. Rychlewski, Chem. Phys. Lett. 1999, 304, 293

45 W. Cencek and J. Rychlewski, Chem. Phys. Lett., 2000, 320, 549.

46 R. Pauncz, Spin Eigenfunctions, Plenum, NY, 1979.

47 D. B. Kinghorn and L. Adamowicz, J. Chem. Phys., 1997, 106, 4589.

48 K. R. Lykke, K. K. Murray and W. C. Lineberger, Phys. Rev., 1991, A43, 6104

49 W. F. Drake, Nucl. Instrum. Methods Phys. Res., Sect. B, 1988 , 31, 7 .

50 D. B. Kinghorn and L. Adamowicz, J. Chem. Phys., 1997, 106, 8760 .

51 W. Kolos and L. Wolniewicz, J. Chem. Phys., 1965, 43, 2429

52 S. Bubin and L. Adamowicz, J. Chem. Phys., 2003, 118(7), 3079.

53 D. B. Kinghorn and L. Adamowicz, J. Chem. Phys., 2000, 113, 4203 .

54 L. C. Biedenharn and J. D. Louck, Angular Momentum in Quantum Physics. Theory and Application, Encyclopedia of Mathematics and Its Applications, Addison-Wesley, Reading, MA, 1981.

55 K. Varga and Y. Suzuki, Phys. Rev. C, 1995, 52, 2885.

56 K. Varga and Y. Suzuki, Few-Body Syst., 1998, 24, 81.

57 Y. Suzuki and K. Varga, Stochastic Variational Approach to Quantum Mechanical Few-Body Problems, Springer-Verlag, Berlin, 1998.

58 M. Cafiero and L. Adamowicz, Chem. Phys. Lett., 2001, 335, 404

59 M. Tachikawa, K. Mori, K. Suzuki and K. Iguchi, Int. J. Quantum Chem., 1998, 70, 491.

60 M. Tachikawa, K. Mori, H. Nakai and K. Iguchi, Chem. Phys. Lett., 1998, 290, 427.

61 H. Nakai, K. Sodeyama and H. Hoshino, Chem. Phys. Lett., 2001, 345, 118

62 H. Nakai, Int. J. Quantum Chem., 2002, 86, 511.

63 A. L. Sobolewski and L. Adamowicz, Chem. Phys., 1995, 193, 67.

64 A. L. Sobolewski and L. Adamowicz, J. Chem. Phys., 1995, 102 5708 .

65 A. L. Sobolewski and L. Adamowicz, Chem. Phys. Lett., 1995 234, 94.

66 A. L. Sobolewski and L. Adamowicz, J. Phys. Chem., 1995, 99, 14277.

67 A. L. Sobolewski and L. Adamowicz, J. Phys. Chem., 1996, 100, 3933.

68 A. L. Sobolewski and L. Adamowicz, Chem. Phys. Lett., 1996 252, 33.

69 A. L. Sobolewski and L. Adamowicz, Chem. Phys., 1996, 213, 193.

70 M. Forés, M. Duran, M. Solá and L. Adamowicz, J. Phys. Chem. $A, 1999, \mathbf{1 0 3}, 4413$.

71 M. Forés, M. Duran, M. Solá and L. Adamowicz, J. Comput. Chem., 2000, 21, 257.

72 M. Forés and L. Adamowicz, J. Comput. Chem., 1999, 20, 1422.

73 T. W. J. Whiteley, A. J. Dobbyn, J. N. L. Connor and G. C. Schatz, Phys. Chem. Chem. Phys., 2002, 2, 529.

74 A. Carrington and R. A. Kennedy, Gas Phase Ion Chemistry, ed. M. T. Bowers, Academic Press, New York, vol. 3, p. 393.

75 I. Forster and C. Kesselman, The Grid: Blueprint for a New Computing Infrastructure, Morgan-Kaufmann, San Francisco, 1999.

76 L. Wolniewicz, J. Chem. Phys., 1995, 103, 1792.

77 D. M. Bishop and L. M. Cheung, Phys. Rev. A, 1977, 18 1846.

78 B. Chen and J. B. Anderson, J. Chem. Phys., 1995, 102, 2802. 